\title{
Rheumatic fever revisited
}

Madeleine W. Cunningham

In their Perspectives article (Tandon, $\mathrm{R}$. et al. Revisiting the pathogenesis of rheumatic fever and carditis. Nat. Rev. Cardiol. 10, 171-177 [2013]), ${ }^{1}$ Tandon et al. provide a very thoughtful analysis and review of the pathogenesis of acute rheumatic fever (ARF) and rheumatic heart disease (RHD). Their views challenge the role of molecular mimicry in rheumatic carditis. For years, two groups of thought have existed, one proposing molecular mimicry between streptococcus and the heart, and the other proposing collagen-mediated disease in the valve. Both could be correct and are supported by data indicating elevation of anticollagen and anti-cardiac-myosin antibody responses in rheumatic carditis. ${ }^{2}$ These two hypotheses are important, but one hypothesis does not exclude the other. Most autoimmune diseases involve more than one autoantigen, ${ }^{3}$ and both cardiac myosin and collagen are autoantigens in $\mathrm{RHD},{ }^{2}$ and one might precede the other.

ARF and RHD develop over time from multiple streptococcal infections ${ }^{4}$ leading to the production of IgG autoantibodies of relatively high avidity. This pathophysiology does not occur as a result of gross immaturity of the immune system, as suggested by Tandon and colleagues, but because of the continual streptococcal infections that maintain the germinal centre reaction and affinity maturation of antibody. Earlier this year, Zhang et al. proposed that antibody feeds back and stops the germinal centre reaction in the normal immune response. ${ }^{5}$ However, in ARF and RHD, high levels of immune complexes would capture antibodies of higher affinity and allow the germinal centres to continue to feed highaffinity autoantibody specificities into the blood, which would eventually cause damage to the valve. When levels of antibodies against streptococcal group A carbohydrate continue to elevate during ARF and RHD, prognosis is poor. Only upon valve replacement do the levels of autoantibody against the valve and group A carbohydrate subside concomitantly with healing and restoration of heart function. ${ }^{6}$ Anti-group A carbohydrate antibodies have long been recognized to bind to human heart valves.?

Mimicry of streptococcal and host antigens allows antibodies to initially attack the endothelial surface of the valve, leading to verrucae and oedema, with vascular cell adhesion protein 1 (VCAM-1) activation on endothelium, so that the immune-privileged valve is compromised and T cells can infiltrate. ${ }^{8}$ Oedema and stretching of the chordae tendinae lead to the first stage of rheumatic carditis. ${ }^{9,10}$ Once the valve is initially damaged at the endocardium, antibodies against collagen-generated from exposure to either collagen released from injured valve, collagen bound to the streptococcus, or both-can deposit in the valve and cause inflammation. Anticollagen antibody induction in the absence of collagen release from the valve might require the attachment of collagen to the streptococcal $\mathrm{M}$ protein to initially induce an immune response to collagen. The valve is immune-privileged, and only when the integrity of the endothelium is broken by activation can the valve become vulnerable to the power of the immune system. ${ }^{8}$ This phenomenon is evident in the scarring and neovascularization of the valve and the explosion of valvular heart disease with every new group A streptococcal infection.

Although cardiac myosin is not present in the valve, it does surround the valve in papillary muscle where Aschoff bodies are often found in subendothelium. ${ }^{8}$ Cardiac myosin was identified on the basis of reports that antistreptococcal antibodies reacted with myocardium, ${ }^{11}$ but the target for these antibodies on the valve was the endothelium. ${ }^{12}$ The basis of this molecular mimicry between myocardium and valve is the shared alpha-helical protein sequences and glycosylated proteins. ${ }^{7,13}$ Anti-cardiacmyosin responses are directly linked with responses against the group A carbohydrate epitope $\mathrm{N}$ acetyl- $\beta$-D glucosamine, ${ }^{13,14}$ which target the valve endothelial surface and laminar basement membrane.

The development of the anticollagen antibody response might be expected against a damaged valve that released collagen upon injury. T cells infiltrating the valves have not been linked to collagen recognition, but to responsiveness against streptococcal M proteins and alpha-helical proteins present in rheumatic valves. ${ }^{15,16}$ The role of valve injury and release of collagen might be important in the development of an autoantibody response against collagen within the valve, but the mechanisms by which these autoantibodies might attack the valve are not known. Multiple autoantibodies against multiple cardiac antigens are likely to be a hallmark of ARF and RHD; collagen has always been one piece of the puzzle, but not all of it.

Department of Microbiology and Immunology, University of Oklahoma Health Sciences Centre, Room 217, 975 NE $10^{\text {th }}$ Street, Oklahoma City, OK 73104, USA. madeleine-cunningham@ouhsc.edu

\section{Acknowledgements}

Research funding to $\mathrm{M}$. W. Cunningham is acknowledged as grants HL35280 and HL56267 from the National Heart, Lung and Blood Institute and funding from the Oklahoma Centre from the Advancement of Science and Technology, all in the USA.

\section{Competing interests}

M. W. Cunningham declares associations with the following company: Moleculera Labs. See the article online for full details of the relationship.

1. Tandon, R. et al. Revisiting the pathogenesis of rheumatic fever and carditis. Nat. Rev. Cardiol. 10, 171-177 (2013).

2. Martins, T. B. et al. Comprehensive analysis of antibody responses to streptococcal and tissue antigens in patients with acute rheumatic fever. Int. Immunol. 20, 445-452 (2008).

3. Arbuckle, M. R. et al. Development of autoantibodies before the clinical onset of systemic lupus erythematosus. N. Engl. J. Med. 349, 1526-1533 (2003).

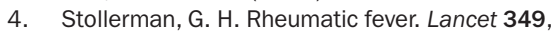
935-942 (1997).

5. Zhang, Y. et al. Germinal center B cells govern their own fate via antibody feedback. J. Exp. Med. 210, 457-464 (2013).

6. Dudding, B. A. \& Ayoub, E. M. Persistence of streptococcal group A antibody in patients with rheumatic valvular disease. J. Exp. Med. 128, 1081-1098 (1968).

7. Goldstein, I., Halpern, B. \& Robert, L. Immunological relationship between streptococcus A polysaccharide and the structural glycoproteins of heart valve. Nature 213, 44-47 (1967). 


\section{CORRESPONDENCE}

8. Roberts, S. et al. Immune mechanisms in rheumatic carditis: focus on valvular endothelium. J. Infect. Dis. 183, 507-511 (2001).

9. Williams, R. V., Minich, L. L., Shaddy, R. E., Veasy, L. G. \& Tani, L. Y. Evidence for lack of myocardial injury in children with acute rheumatic carditis. Cardiol. Young 12, 519-523 (2002).

10. Ellis, N. M. et al. Priming the immune system for heart disease: a perspective on group $A$ streptococci. J. Infect. Dis. 202, 1059-1067 (2010).

11. Krisher, K. \& Cunningham, M. W. Myosin: a link between streptococci and heart. Science 227, 413-415 (1985).
12. Galvin, J. E., Hemric, M. E., Ward, K. \& Cunningham, M. W. Cytotoxic mAb from rheumatic carditis recognizes heart valves and laminin. J. Clin. Invest. 106, 217-224 (2000).

13. Galvin, J. E. et al. Induction of myocarditis and valvulitis in Lewis rats by different epitopes of cardiac myosin and its implications in rheumatic carditis. Am. J. Pathol. 160, 297-306 (2002).

14. Malkiel, S., Liao, L., Cunningham, M. W. \& Diamond, B. T-cell-dependent antibody response to the dominant epitope of streptococcal polysaccharide, $\mathrm{N}$-acetyl- glucosamine, is cross-reactive with cardiac myosin. Infect. Immun. 68, 5803-5808 (2000).

15. Fae, K., Kalil, J., Toubert, A. \& Guilherme, L. Heart infiltrating $T$ cell clones from a rheumatic heart disease patient display a common TCR usage and a degenerate antigen recognition pattern. Mol. Immunol. 40, 1129-1135 (2004).

16. Ellis, N. M., Li, Y., Hildebrand, W., Fischetti, V. A \& Cunningham, M. W. T cell mimicry and epitope specificity of crossreactive T cell clones from rheumatic heart disease. J. Immunol. 175, 5448-5456 (2005) 